\title{
Surgimiento y evolución de la cultura Manteña- Guancavilca: reflexiones acerca de los cambios y continuidades en la costa del Ecuador prehispánico
} Émergence et évolution de la culture Manteña-Guancavilca : réflexions autour des changements et des continuités sur la côte de l'Équateur préhispanique Emergence and evolution of the Manteño-Guancavilca culture: Comments about changes and continuities on prehispanic Ecuadorian coast

\section{Anne Touchard-Houlbert}

\section{OpenEdition}

Journals

Edición electrónica

URL: http://journals.openedition.org/bifea/1750

DOI: $10.4000 /$ bifea. 1750

ISSN: 2076-5827

Editor

Institut Français d'Études Andines

Edición impresa

Fecha de publicación: 1 diciembre 2010

Paginación: 551-561

ISSN: 0303-7495

Referencia electrónica

Anne Touchard-Houlbert, «Surgimiento y evolución de la cultura Manteña-Guancavilca: reflexiones acerca de los cambios y continuidades en la costa del Ecuador prehispánico », Bulletin de l'Institut français d'études andines [En línea], 39 (3) | 2010, Publicado el 01 junio 2011, consultado el 08 diciembre 2020. URL : http://journals.openedition.org/bifea/1750 ; DOI : https://doi.org/10.4000/bifea. 1750

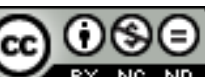

Les contenus du Bulletin de l'Institut français d'études andines sont mis à disposition selon les termes de la licence Creative Commons Attribution - Pas d'Utilisation Commerciale - Pas de Modification 4.0 International. 


\title{
Surgimiento y evolución de la cultura Manteña-Guancavilca: reflexiones acerca de los cambios y continuidades en la costa del Ecuador prehispánico
}

\author{
Anne Touchard-Houlbert*
}

\section{Resumen}

Este artículo aborda los problemas de las rupturas y continuidades en la cronología de la costa ecuatoriana con un estudio global de la cultura Manteña-Guancavilca, la cual se ha desarrollado en el litoral ecuatoriano durante el último periodo precolonial (650-1532 d. C.). De hecho, con el estudio de la ocupación humana, de la variabilidad de los tipos arquitectónicos y del complejo cerámico, se intenta evaluar las diferentes fuentes que permiten entender los cambios culturales en esta área.

Palabras clave: arqueología, Ecuador, Periodo de Integración, Manteña-Guancavilca, arquitectura, prácticas funerarias, cerámica, señorio

\section{Émergence et évolution de la culture Manteña-Guancavilca :} réflexions autour des changements et des continuités sur la côte de l'Équateur préhispanique

\section{Résumé}

À travers une étude globale de la société Manteña-Guancavilca qui occupa le littoral équatorien au cours de la dernière période précoloniale (650-1532 apr. J.-C.). Cet article aborde la problématique des ruptures et des continuités dans la chronologie de la côte équatorienne. En effet, l'étude de 
I'occupation humaine, des différents types architecturaux et du complexe céramique, nous renseigne sur les différentes sources de changement culturel dans une même région.

Mots clés : archéologie, Équateur, Période d'Intégration, Manteña-Guancavilca, architecture, pratiques funéraires, céramique, señorio

\title{
Emergence and evolution of the Manteño-Guancavilca culture: Comments about changes and continuities on prehispanic Ecuadorian coast
}

\begin{abstract}
Through a general study of the Manteño-Guancavilca culture along the Ecuadorian littoral during the final pre-colonial period (650-1532 AD.), in this article we focus the problems of the ruptures and continuities in the chronology of the Ecuadorian coast. Indeed, through the study of the human occupation, of the variability of architectural types and the ceramic complex, we evaluate different sources for understanding the way in which cultural changes were producted in this area.
\end{abstract}

Key words: archaeology, Ecuador, Integration Period, Manteño-Guancavilca, architecture, funerary pratices, ceramics, señorio

\section{INTRODUCCIÓN}

El estudio que se ha realizado trata de entender cómo y por qué han ocurrido los cambios y continuidades a lo largo de la cronología de la época prehispánica (cambios en los estilos cerámicos, los modos arquitectónicos y la organización del espacio...) en la costa ecuatoriana. Para eso, se ha tratado, enfocándose en una cultura cuya cronología no está bien definida: la Manteña-Guancavilca (6501532 d. C.), de identificar las supervivencias de las culturas pasadas así como las innovaciones propias de esa cultura y, de esa forma, entender su evolución. El objetivo es establecer una secuencia cronológica de la cultura ManteñaGuancavilca y entender así cómo aparece, cómo funciona y cómo desaparece a la llegada de los españoles. En este artículo, no vamos a tratar del periodo de contacto con los conquistadores ni de la destrucción de esa sociedad, pero vamos a intentar responder, en la medida de lo posible, a preguntas «sencillas»: ¿̇dónde, cuándo, cómo y por qué?

\section{LA OCUPACIÓN TERRITORIAL MANTEÑA-GUANCAVILCA}

Para entender cómo la cultura Manteña-Guancavilca se desarrolló, es importante definir su entorno geográfico e identificar algunos indicios que nos ayuden a

1 Ese artículo, se nutre de los resultados de la tesis doctoral del autor, defendida el 15 de enero del 2010 en La Sorbona (Paris, Francia) y en los de las excavaciones que llevó a cabo el proyecto Manabí Central en el yacimiento de Japotó, Manabí desde 2004 y a las cuales pudo participar el autor. 
entender los diferentes movimientos de población, en la totalidad de la fase de ocupación (650-1532 d. C.).

Para esto, ha sido establecido un mapa arqueológico (fig. 1), basándose en la bibliografía arqueológica, los informes de prospecciones y de excavaciones, así como en informes de arqueología de rescate. Lamentablemente, no es posible ver una localización completa de la ocupación humana dado que los 161 sitios arqueológicos registrados están concentrados en la franja marítima.

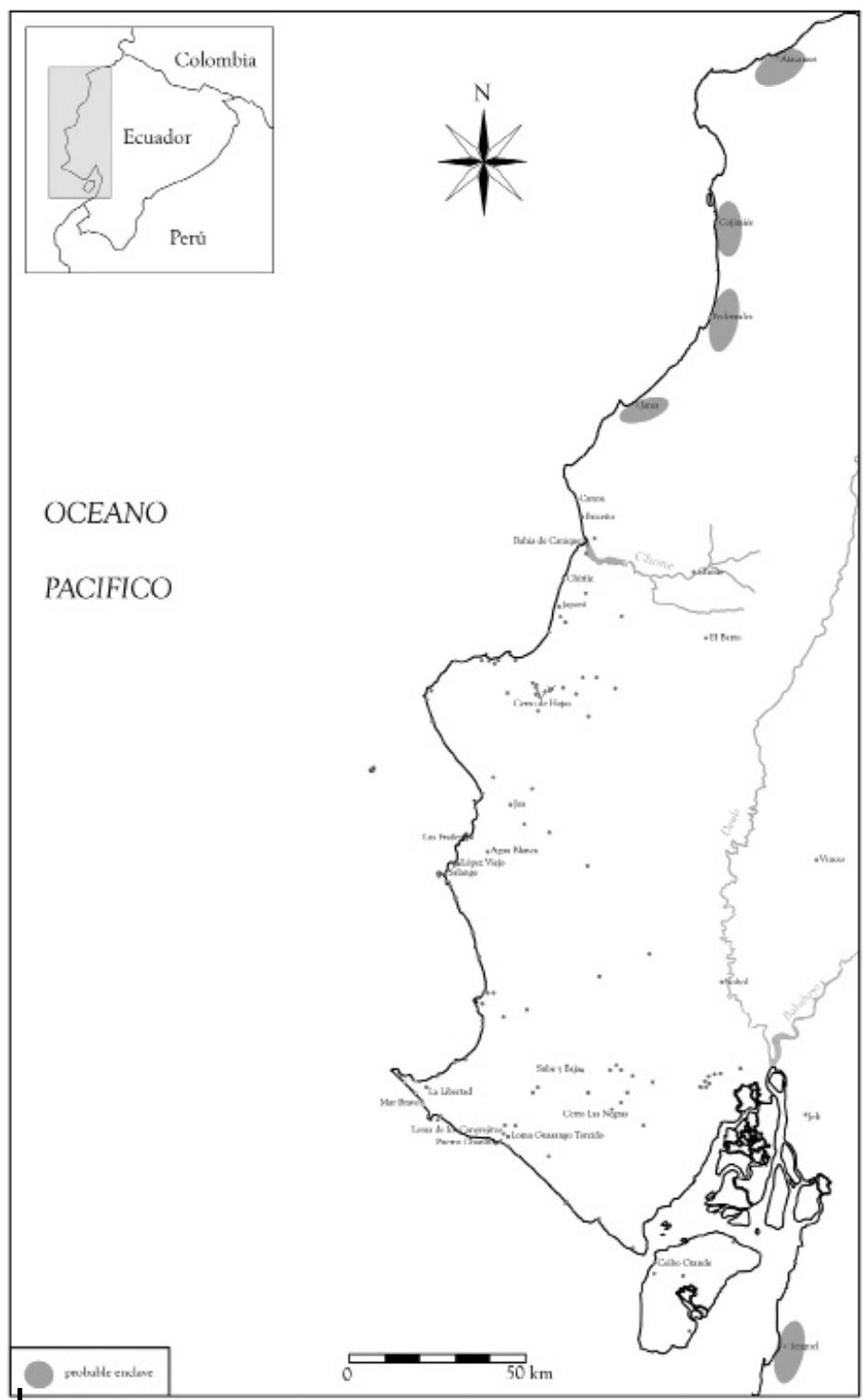

Figura 1 - Mapa de los sitios Manteño-Guancavilca localizados 


\section{1. Las fronteras de la confederación Manteña-Guancavilca}

De manera general, se toma en cuenta la visión global conocida hasta ahora según la cual las fronteras de la confederación² Manteña-Guancavilca están localizadas al norte en el rio Chone, y al sur en el golfo de Guayaquil. Sin embargo, algunos sitios como Tengel o Jeli han sido registrados como Manteña-Guancavilca y se puede pensar que han habido variaciones de las fronteras según las épocas. La frontera oriental no está claramente determinada ya que se han realizado escasos estudios en la zona del piedemonte andino. Los sitios más al este son los de Chone y Nobol. Se incorporan los sitios de El barro y Vinces, donde se han registrado piezas Manteña-Guancavilca, pero donde no hay ocupación averiguada. Otros objetos han sido descubiertos en varios sitios al norte de Canoa (Jama, Pedernales, Cojimies y Atacames). Las localizaciones observadas en los registros, tenderían a desplazar más la frontera hacia el norte. No obstante, estos objetos no son típicos de esta cultura, sino que indican claramente semejanzas, permitiendo apreciar la evolución de una forma a otra (p.ej.: los pedestales antropomórficos).

Según los datos etnohistóricos, localidades como Atacames (Tacamez) se consideran como lugares colocados bajo los auspicios de uno de los hombres de poder (o Señor) y más concretamente el «señor de Salangome» (Jerez, 1972 [1534]). En este texto, se habla de las ciudades o pueblos sometidos al Señorio de Salangome. Estas ciudades, fuera del territorio conocido y definido como Manteña-Guancavilca se interpretan como «enclaves» portuarios (Ruiz, 1844). Sin embargo, los vestigios que permiten justificar esta hipótesis son muy dispersos. En efecto, los únicos vestigios excavados en contexto son los provenientes de Atacames (Guinea, 1984). Parece difícil, a partir de estos datos demasiados escasos, establecer la existencia real de un sistema de enclaves en otros territorios. Aunque el concepto de puerto que sirve de escala donde se habrían instalado algunos Manteña-Guancavilca sea muy posible, sería necesario tener más información para determinar el lugar real que este grupo ha poseído en los territorios periféricos.

\section{2. La organización espacial de los sitios arqueológicos}

El mapa arqueológico que se ha podido realizar a partir de estos datos permite definir zonas de asentamientos que, en espera de estudios más detallados, ayudan a entender cómo se distribuyen los yacimientos arqueológicos en el territorio. Por supuesto, se consideran los datos de una manera global pero los pocos lugares con dataciones radiocarbónicas (o al menos su escasa proporción en relación al número total de lugares localizados: 15 sobre 161) no permiten ver de manera diacrónica la evolución de las ocupaciones humanas. Sin embargo se puede tener una idea de ello gracias al estudio de Yann Graber (2008) para la zona de Río Blanco. Se han establecido zonas de instalación: el frente marítimo, el litoral cercano (menos

\footnotetext{
2 Hablamos de confederación en el sentido de grupo de varios señorios regionales.
} 
de $5 \mathrm{~km}$ de la costa), tierradentro y los lugares de montaña, teniendo cada zona probablemente un papel particular en el proceso de producción y circulación de los bienes de las poblaciones locales (Murra, 1975).

\section{3. La distribución diacrónica de los sitios}

Pocos sitios proporcionaron fechas que nos ayuden en definir la ocupación del espacio. En efecto, solo 15 sitios dieron fechas radiocarbónicas que permitieron situarles en la cronología.

La información deja suponer que todo el territorio ha sido ocupado a la vez, de norte a sur. En efecto, se han descubierto algunos elementos Manteña-Guancavilca en los sitios de Japoto, Los Frailes, Loma de los Cangrejitos y Loma de Guasango, lugares cuya ocupación Manteña-Guancavilca se prueba antes del siglo VIII d. C. Es difícil imaginar una progresión lenta de la ocupación (debida a una probable invasión). La única hipótesis que cuadra con este sentido es una invasión por vía marítima (cf. localización de las enclaves en la fig. 1).

\section{LA SECUENCIA CRONOLÓGICA}

La secuencia cronológica pudo realizarse gracias a la revisión de las fechas disponibles, un total de 59 muestras (fig. 2).

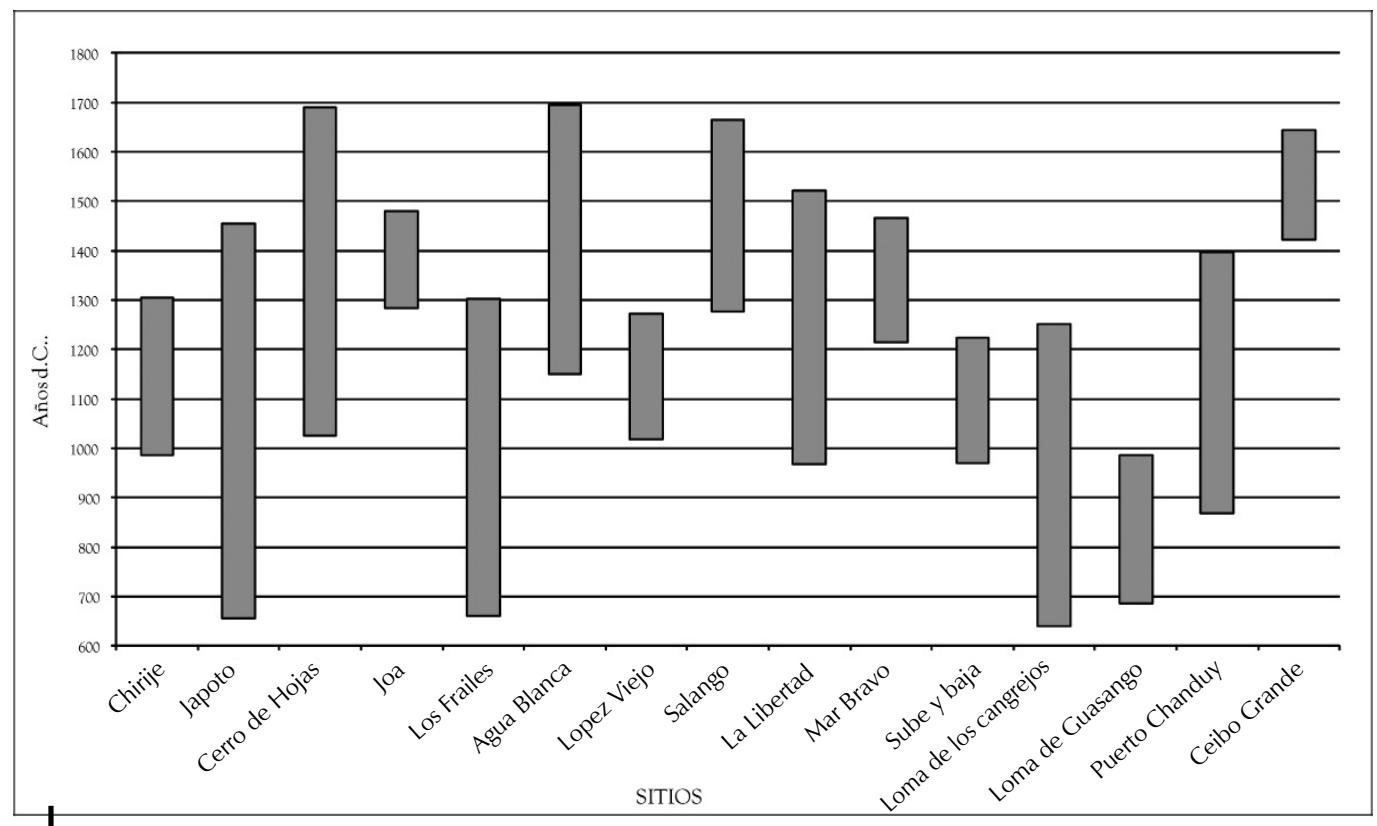

Figura 2 - Representación de las secuencias de ocupación de 15 sitios Manteña-Guancavilca 
Estos datos permiten concluir que hay una occupación homogénea del territorio (en el sentido norte-sur), rechazando así la hipótesis de un modelo de migración expansionista desde el norte o el sur como lo ha imaginado Jijón y Caamaño (1930). Al contrario, esto apoya más bien la hipótesis de una transición desde el periodo del Desarrollo Regional (500 a. C.-500 d. C.) hasta el de Integración con un origen local, y no como resultado de una colonización externa, excepto quizá por el mar aunque no hayan elementos que permitan apoyar esto último.

Es difícil percibir de manera clara cómo se ha producido esta transición y cuáles son sus razones y consecuencias.

No se ha podido demostrar un cambio en el orden social debido a la falta de estudios, especialmente sobre la cultura Bahía que se ha desarrollado en la misma zona (Manabí Central), en un periodo al inmediatamente anterior que se considera aquí.

De la misma manera, se tiene muy poca información arqueológica y especialmente estratigráfica sobre la transición entre las fases Bahía/Guangala y ManteñaGuancavilca. En Japoto, de momento, no se ha podido identificar esta transición que, sin embargo, es muy clara en el sitio OM Jp Lp 140 de Salango (Norton, nd).

\section{LAS HUELLAS DEL PASADO}

A raíz de la carencia de datos de terreno, se utilizará el material cerámico disponible (3 784 piezas de 12 colecciones revisadas) para conocer cuáles son los elementos procedentes de cada una de estas culturas, qué elementos persisten, cuáles desaparecen y qué nuevos elementos resultan, ya sean endógenos o procediendo de las zonas periféricas.

Tres culturas precedieron y coexistieron con la cultura Manteña-Guancavilca: Bahía, Guangala y Chirije. A partir de los elementos descubiertos en el yacimiento de Japoto esto fue lo que salió a la luz:

De la cultura Bahía, se han podido identificar varios elementos (fig. 3):

Las orejeras parecidas a las que se identifican en las figuritas Bahía gigante (fig. 3a).

La decoración bruñido grueso (Estrada, 1962: 145, fig. 44) (fig. 3b).

El tipo Bahía Muescas al reborde (Estrada, 1962: 151, fig. 53) (fig. 3c).

El tipo Bahía sobre amarillo (fig. 3d).

El tipo Bahía calado (fig. 3e).

De la cultura Guangala se pudo reconocer (fig. 4):

La decoración bruñida fina (Masucci, 1992: 520, fig. 38) (fig. 4a).

La decoración, grabada sobre pintura negativa (fig. 4 b).

De la cultura Chririje: lamentablemente, esta cultura aún está mal definida; no obstante en Japoto aparecen varios elementos de este complejo cerámico, como se ve en la figura 5: 


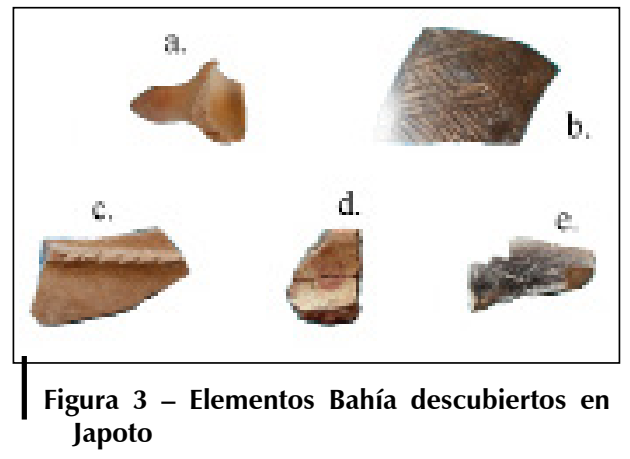

Fotos: A. Touchard-Houlbert

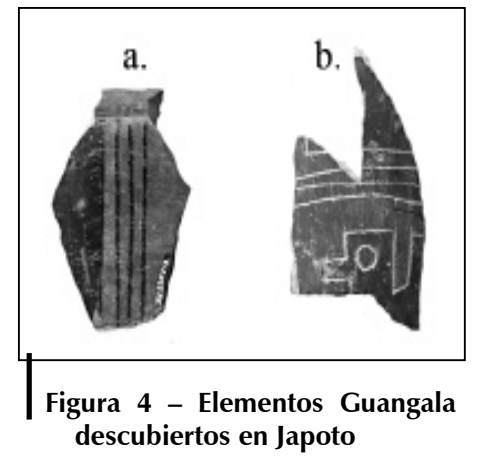

Fotos: A. Touchard-Houlbert

El estilo Chirije estampado dentado en zonas (Estrada, 1962: 132, fig. 66) (fig. 5a).

El rallador punteado (fig. 5b).

No se sabe realmente si el complejo cerámico Chirije corresponde a una transición Bahía/Manteña-Guancavilca o a un verdadero complejo cultural.

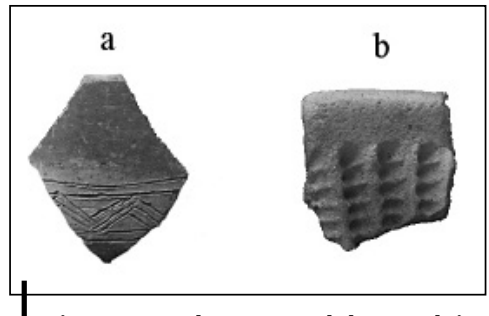

Figura 5 - Elementos del complejo Chirije descubiertos en Japoto

Fotos: A. Touchard-Houlbert

Lamentablemente, las evidencias son escasas pero aunque no permitan aclarar la secuencia ManteñaGuancavilca de manera precisa, por lo menos ayudan a identificar los diferentes elementos estilísticos que han estado presentes en el sitio (fig. 6).

Así, junto a la cultura Manteña-Guancavilca permanecieron elementos estilísticos más antiguos, desaparecieron otros, fuertemente introducidos en el periodo de Desarrollo Regional como los polípodos (Guangala y Bahía) que se hicieron cada vez más raros o el uso de la famosa pintura tricolor Guangala.

Poco a poco, han aparecido nuevos tipos cerámicos (como los tostadores y los barreños), nuevos motivos y representaciones, los cuales supuestamente han cambiado con el tiempo. Sin embargo nos resulta todavía difícil entender la secuencia cerámica Manteña-Guancavilca, porque de un lado nota cierta estandarización de la producción alfarera con la famosa «Manteña negra pulida» pero, al mismo tiempo, se puede identificar una gran variedad de formas, decoraciones, etc.

Tanto en Japoto como en Salango, donde se ha podido revisar gran parte del material, se observó una concentración de ollas globulares (fig. 7a), de tostadores y compoteras (fig. 7b) y de vasijas evertidas con representaciones modeladas en el cuello (fig. 7c). 


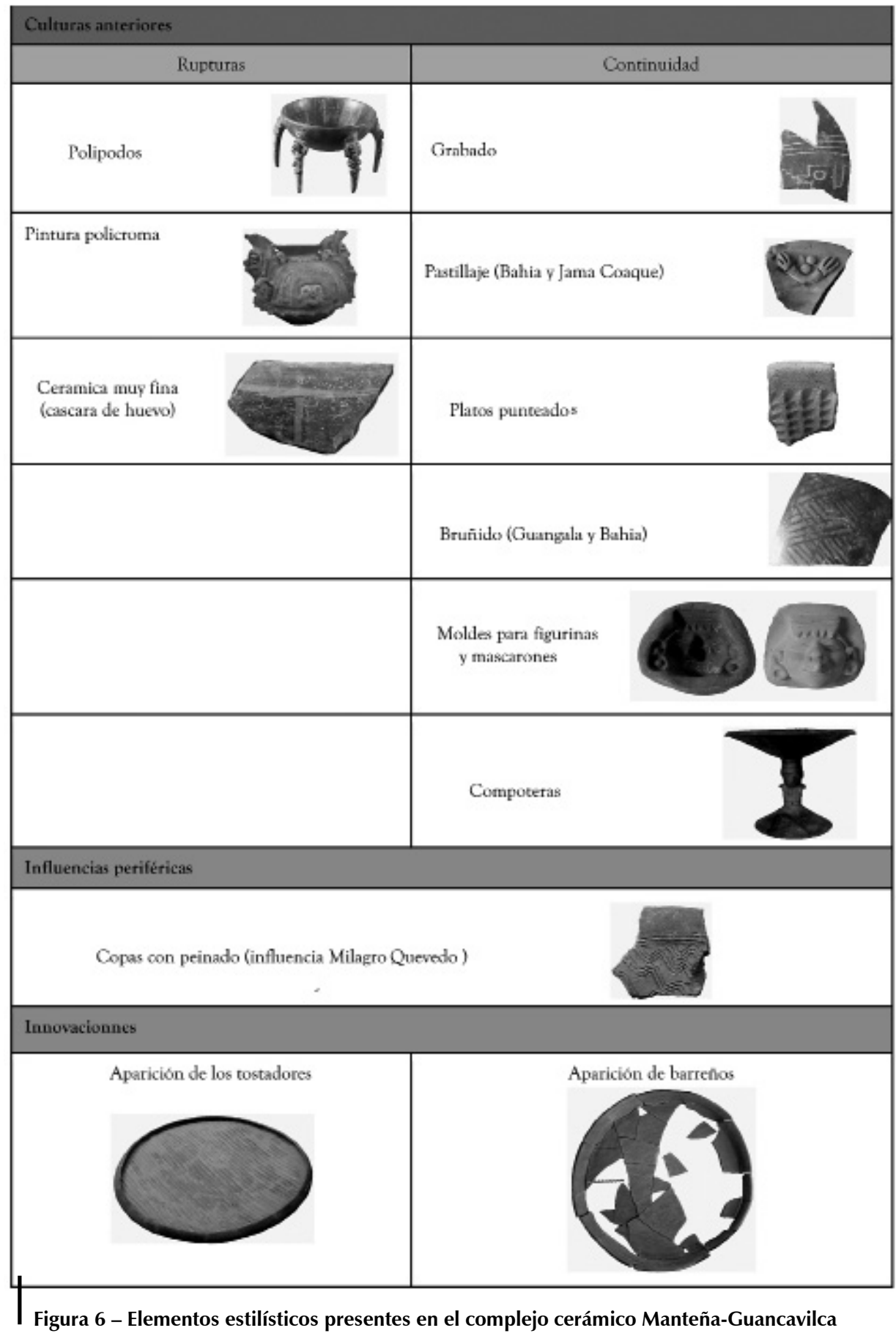

Fotos: A. Touchard-Houlbert 


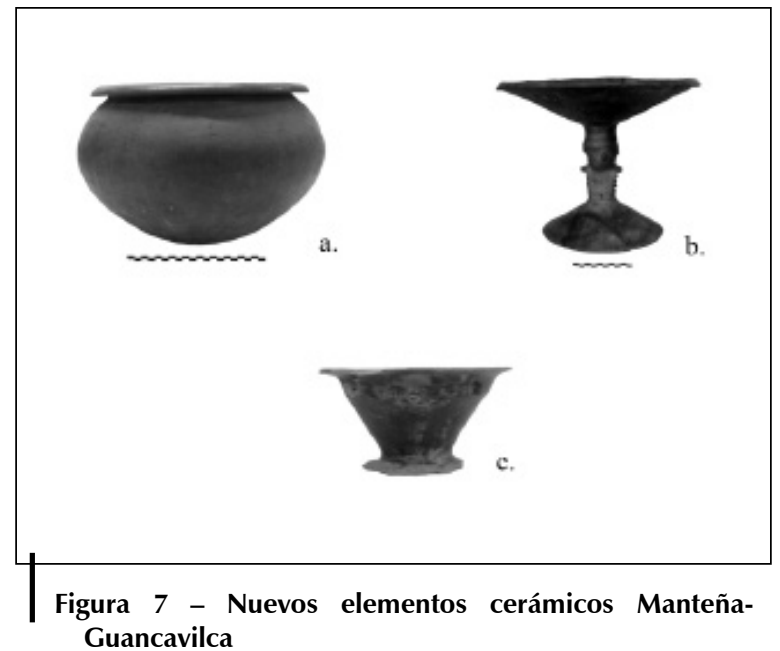

Fotos: A. Touchard-Houlbert

\section{LOS TIPOS DE ASENTAMIENTOS Y EL HIATO DEL 1100}

A lo largo de nuestra investigación, hemos identificado varios tipos sucesivos de asentamientos en toda el área Manteña-Guancavilca. Los primeros asentamientos, al nivel del suelo, fueron seguidos por la erección de tolas (o montículos artificiales), las cuales han funcionado hasta el final del periodo prehispánico.

Por un motivo que todavía no está determinado, pero que supuestamente corresponde a un evento de importancia, aparecen nuevos elementos arquitectónicos alrededor del siglo XII, tales como las estructuras de adobe en Agua Blanca (McEwan, 2003; Piana Bruno \& Marotzke, 1983), en Loma de los Cangrejitos (Marcos, 1973) y en Japoto (ver Guinea este volumen) y las estructuras de piedra en los sitios de altura o del litoral (fig. 8).

Este cambio fuerte durante la fase Manteña-Guancavilca (el evento «1100» como lo hemos llamado) representa quizás una ruptura importante adentro de la sociedad Manteña-Guancavilca, una modificación de la organización sociopolítica y una voluntad de cambiar el modelo de asentamiento, aunque sigan utilizando el sistema de tolas.

Sin embargo, ese cambio en la estrategia arquitectónica no parece observarse en la cerámica, al menos en la que he revisado. Es posible que con más fechas en el sitio de Japoto y un estudio más preciso de la cerámica, logremos establecer una secuencia cerámica que ponga en relación esas etapas constructivas con la tipología cerámica. 


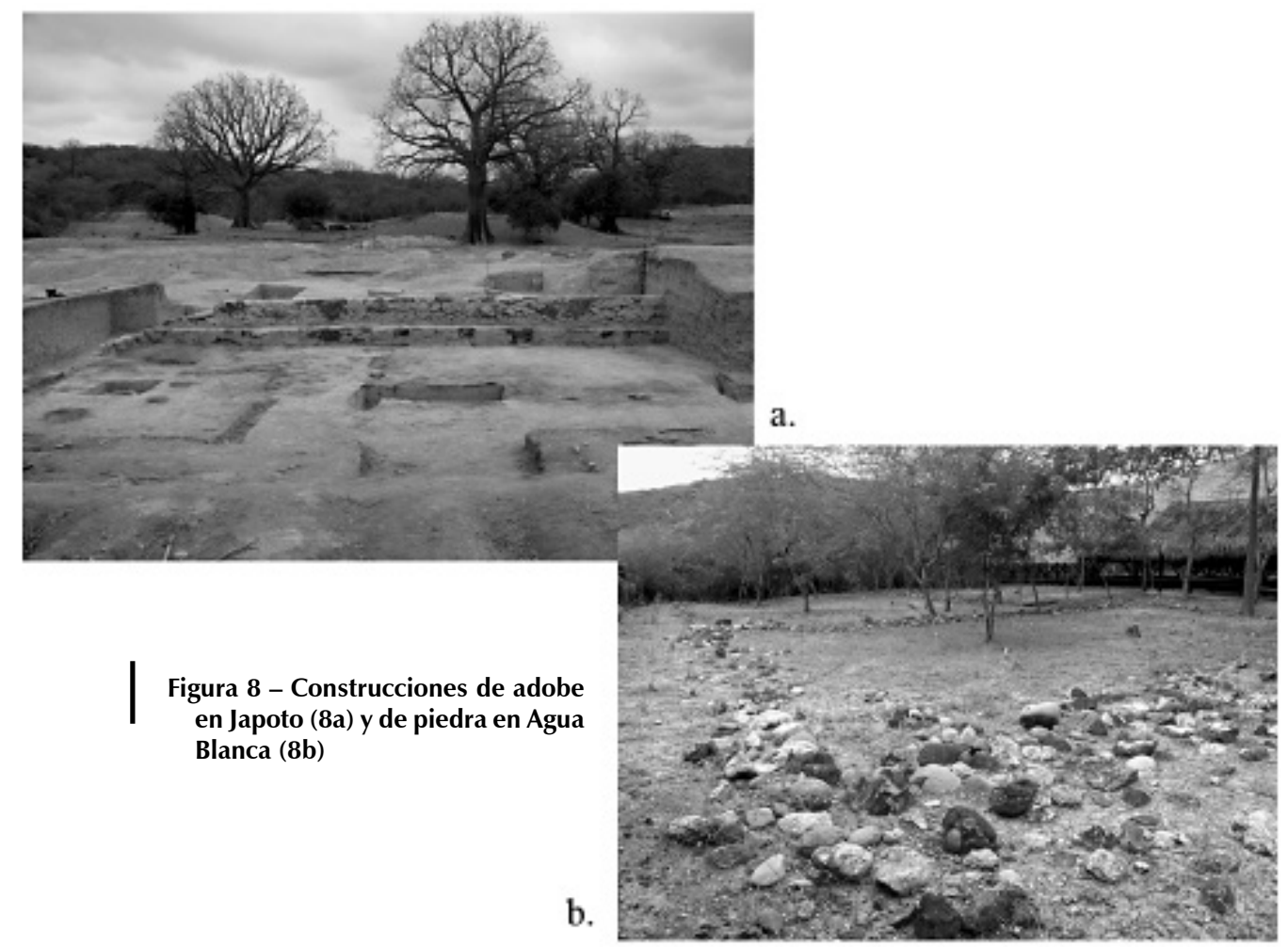

\section{CONCLUSIONES}

En conclusión, podemos decir que este estudio ha permitido establecer que los Manteña-Guancavilca ocupaban el territorio de manera homogénea a lo largo de toda la fase cultural correspondiente.

El estudio preliminar de la cerámica nos indica tanto una continuación en formas y decoraciones de las culturas anteriores como rupturas en cuanto a la utilización de ciertos elementos o motivos decorativos.

La posibilidad de fechar el inicio de la utilización de los adobes y de las piedras como elementos constructivos arquitecturales, permite observar que ocurre un cambio importante alrededor de $1100 \mathrm{~d}$. C., cambio recurrente en toda la zona andina.

Este artículo ha querido brindar nuevos elementos de la secuencia ManteñaGuancavilca, a través del estudio del complejo arquitectural y de la cerámica.

El problema de las transiciones culturales parece ser el mismo en cualquier lugar y sigue siendo difícil identificar los factores originarios de estos cambios. En el caso presente, se van a identificar paulatinamente estos cambios y estas continuidades. 
Es de esperar que nuevos investigaciones permitirán responder a las preguntas que han quedado pendientes.

\section{Referencias citadas}

ESTRADA, E., 1962 - Arqueología de Manabì Central, 205 pp.; Guayaquil: Publicaciones del Museo Vistor Emilio Estrada n. ${ }^{\circ} 7$.

GRABBER, Y., 2008 - Aux origines du «señorio de Salangome». Occupation territoriale préhispanique de la côte sud de Manabí (Équateur). Les nouvelles de l'archéologie. Des mers de glaces à la Terre de Feu, Archéologie Française en Amérique. n. ${ }^{\circ} 111$ 112, Avril 2008: 59-65; París: Éditions de la maison des sciences de l'homme. Éditions Errance.

GUINEA BUENO, M., 1984 - Patrones de asentamiento en la arqueología de Esmeraldas (Ecuador). Memorias de la Misión Arqueológica Española en el Ecuador, vol. 8; Madrid: Ministerio de Asuntos Exteriores.

JEREZ, F. 1972 [1534] - Verdadera Relación de la Conquista del Perú; Madrid: Historia 16. JIJÓN y CAAMAÑO, J., 1930 - Una gran marea cultural en el noroeste de Sudamérica. Journal de la Société des Américanistes, n. ${ }^{\circ} 22:$ 107-197; París.

MARCOS, J., 1973 - The necropolis at the Loma de los Cangrejitos, Chanduy valley; : Department of Anthropology, University of Illinois.

MASUCCI, M., 1992 - Ceramic change in the Guangala Phase, Southwest Ecuador: A typology and Chronology; Southern Methodist Universtity. Ph. Dissertation.

McEWAN, C., 2003 - And the sun sits in his seat: creating social order in Andean culture; Urbana-Champaingn: University of Illinois. Ph.D. Dissertation.

MURRA, J. V., 1975 - El control vertical de un máximo de pisos ecológicos en la economía de las sociedades andinas. In: Formaciones económicas y políticas en el mundo andino: 59-115; Lima: Instituto de Estudios Peruanos.

NORTON, P., n.d. - Journal des fouilles de Salango.

PIANA BRUNO, L. \& MAROTZKE LETZEL, H., 1983 - Las estructuras de Agua Blanca. Un complejo Mantense. Rapport manuscrit déposé à I'Instituto Nacional de Patrimonio Cultural de Guayaquil.

RUIZ, B. (Relacion de Juan Sámano), 1844 - Relación (1526). In: Relación de los Primeros Descubrimientos de Francisco Pizarro y Diego de Almagro: 193-201; Madrid: Imprenta de la Viuda de Calero, 1844. T. V. Colección de Documentos Inéditos para la Historia de España (Relación sacada del Códice CXX de la Biblioteca Imperial de Viena).

TOUCHARD-HOULBERT, A., 2010 - Rupture et continuité dans la chronologie de la côte équatorienne : Réflexions autour de la société Manteña-Guancavilca; París: Université Paris 1 Panthéon-Sorbonne. Thèse de doctorat. 